\title{
Herpes simplex encephalitis treated with acyclovir: diagnosis and long term outcome
}

\author{
N McGrath, N E Anderson, M C Croxson, K F Powell
}

\begin{abstract}
Objectives- The frequency and characteristics of the long term sequelae of herpes simplex encephalitis were assessed after treatment with acyclovir.

Methods-Patients were included if they were treated with acyclovir and the diagnosis of herpes simplex encephalitis was confirmed by culture of herpes simplex virus (HSV) from the brain, an increase in the CSF HSV antibody titre, or detection of HSV deoxyribonucleic acid in the CSF. Each patient's medical records were reviewed and surviving patients were interviewed and examined.

Results-A diagnosis of herpes simplex encephalitis was confirmed in 42 patients. Five patients $(12 \%)$ died in the first month. Three patients $(7 \%)$ had severe neurological sequelae and died after a longer interval. All but one of the 34 surviving patients had neurological symptoms, an abnormal neurological examination, or both. Twenty patients $(48 \%)$ performed everyday activities as well as before herpes simplex encephalitis; nine patients $(21 \%)$ were living independently, but were functioning at a lower level than before the illness; and five patients (12\%) had a severe neurological deficit. Twenty nine of the 34 survivors were assessed six months to 11 years after herpes simplex encephalitis. The most common long term symptoms were memory impairment $(69 \%)$, personality and behavioural abnormalities (45\%), and epilepsy (24\%). Short term memory impairment $(70 \%)$, anosmia $(65 \%)$, and dysphasia $(41 \%)$ were the most common signs.

Conclusions-Although acyclovir has reduced the mortality of herpes simplex encephalitis, $30 \%$ of this group of patients either died or had a severe neurological deficit. The other $70 \%$ of the patients regained independence in activities of daily living, but most of these people had persistent neurological symptoms, signs, or both.
\end{abstract}

(F Neurol Neurosurg Psychiatry 1997;63:321-326)

Keywords: herpes simplex, encephalitis, acyclovir, polymerase chain reaction

Before effective antiviral treatment was available, the mortality rate of herpes simplex encephalitis was about $70 \%,{ }^{1-3}$ and most survivors had severe neurological deficits. ${ }^{1-10}$ In randomised trials acyclovir reduced the six month mortality rate to about $20 \%$; one quarter to one third of patients required long term supportive care and about $50 \%$ returned to normal life. ${ }^{112}$ Two studies of small groups of long term survivors of herpes simplex encephalitis suggested that sequelae after treatment with acyclovir were more common than implied by the randomised trials. ${ }^{1314}$ Interpretation of many of the other reports on the long term outcome of herpes simplex encephalitis is limited by lack of a definitive diagnosis, ineffective treatment, or selection of patients with known neuropsychological sequelae. We reviewed the long term outcome in an unselected group of 42 patients with herpes simplex encephalitis who had been treated with acyclovir. We also reviewed our experience with the polymerase chain reaction assay for herpes simplex virus (HSV) DNA in the diagnosis of herpes simplex encephalitis and the reasons for misdiagnosis.

\section{Methods}

Patients were traced from records kept by the histopathology and virology departments at Auckland Hospital. They were included if they were treated with acyclovir and the diagnosis of herpes simplex encephalitis was confirmed by one or more of the following: (1) culture of HSV from the brain; (2) a fourfold or greater rise in the HSV antibody titre in the CSF; (3) detection of HSV DNA in CSF. Acyclovir has been available in New Zealand since 1983. Neonatal patients were excluded.

The virology laboratory has received CSF specimens from patients with suspected herpes simplex encephalitis from other parts of New Zealand; these patients were included if they met the inclusion criteria. A few of these patients were managed in small hospitals which did not have CT, EEG, or neurology services. Most hospitals in New Zealand did not provide MRI at the time of this study.

Aliquots of CSF were tested for HSV DNA using the polymerase chain reaction ${ }^{15}$ and for HSV antibodies with a complement fixation assay. The virus was identified by restriction endonuclease digestion of the amplified product. ${ }^{15}$ When a brain biopsy was obtained, a portion was homogenised and inoculated into human diploid fibroblast cell cultures, which were observed for four weeks before being discarded as negative. Other portions were fixed for histopathology and electron microscopy.

Each patient's hospital notes were reviewed. The symptoms, signs, initial clinical diagnosis, investigations, time from the onset of symp- 
toms to the start of treatment, reasons for delay in starting treatment, duration of treatment, and outcome at discharge from hospital were recorded. Whenever possible, we reviewed the CT but EEGs were not reviewed.

Each available surviving patient was interviewed and examined by NM or NEA. The neurological examination included the "mini mental state examination". ${ }^{16}$ Short term memory was tested by asking the patient to memorise and recall a list of five items after five minutes. Recall of four or five items was considered to be normal, two or three items mildly abnormal, and less than two items severely abnormal.

Handicap and quality of life were assessed by questioning the patient and relatives about performance at work, at school, and in activities of daily living. Information about patients who were not available for an interview and examination was obtained by telephone interview, from other hospital records, and from the patients' general practitioners. $\mathrm{Pa}$ tients who died after the first month were graded on the best level of function achieved after the acute illness. Handicap and quality of life were graded with the Glasgow outcome scale: ${ }^{17} 18$ death within the first month; $2=$ vegetative state; $3=$ severe disability (conscious, needing assistance for some activities of daily living; included patients who were independent in most activities of daily living, but required constant supervision because they were unable to organise their daily activities or leave their home without supervision); $4=$ moderate disability (neurological or intellectual impairment, but independent; some previous occupational and social activities were no longer possible); $5=$ good recovery (leading an independent life, with or without minimal neurological impairment). To assess factors that affected long term morbidity, patients who had a poor outcome (Glasgow outcome score $1,2,3)$ were compared with patients with a good outcome (Glasgow outcome score 4, 5). Twenty two long term survivors also had a neuropsychological assessment which will be described in a separate paper.

Data were analysed using the $t$ test for independent samples and the $\chi^{2}$ test for observed frequencies.

\section{Results}

Forty two patients, 27 females and 15 males, with herpes simplex encephalitis were treated with acyclovir from 1983 to 1995. Eleven patients $(26 \%)$ were admitted to Auckland Hospital, seven $(17 \%)$ to other hospitals in Auckland, and $24(57 \%)$ to hospitals outside Auckland. The diagnosis was confirmed by detection of HSV DNA in the CSF in 36 patients, culture of HSV from the brain in four patients, and a significant rise in the CSF HSV antibody titre in two patients. Of the 36 patients with HSV DNA in the CSF, two also had a brain biopsy which grew HSV and in 14 patients antibody to HSV was present in the CSF.
Table 1 Main presenting symptoms, signs, and investigations before treatment with acyclovir $(n=42)$

\begin{tabular}{lr}
\hline & $n(\%)$ \\
\hline Symptoms: & $31(74)$ \\
Headache & $28(67)$ \\
Confusion & $25(60)$ \\
Nausea, vomiting & $21(50)$ \\
Fever & $21(50)$ \\
Seizures & $19(45)$ \\
Drowsiness & $12(28)$ \\
Anorexia & $10(24)$ \\
Abnormal behaviour & $10(24)$ \\
Lethargy & $9(21)$ \\
Cough, sore throat, coryza & \\
Signs: & $41(98)$ \\
Fever & $38(90)$ \\
Abnormal mental state & $23(55)$ \\
Meningism & $14(33)$ \\
Hemiparesis & $12(28)$ \\
Dysphasia & $9(21)$ \\
Homonymous hemianopia & $8(19)$ \\
Minor upper motor neuron signs & $4(9)$ \\
Hemisensory signs & \\
Investigations: & $25 / 38(66)$ \\
Abnormal CT & $38(90)$ \\
CSF pleocytosis & $32(76)$ \\
Increased CSF protein & $36 / 36(100)$ \\
Positive assay for HSV DNA in CSF & $16 / 21(76) \dagger$ \\
HSV antibody present in CSF & $26 / 28(93)$ \\
Abnormal EEG & \\
\hline
\end{tabular}

* Extensor plantar reflex, hyperreflexia.

t CSF samples 10 days or more after the first lumbar puncture were not tested for HSV antibody in the other 21 patients.

CLINICAL FEATURES OF THE ACUTE ILLNESS

The age at presentation ranged from three months to 91 years (median 47 years; mean 43 years); three patients were less than 12 months old. The duration of symptoms before admission was between one and 30 days (median four days, mean five days); all but one patient had symptoms for 10 days or less. Headache, confusion, nausea, fever, seizures, and drowsiness were the most common presenting symptoms (table 1). Most patients were confused, drowsy, or unconscious, and focal neurological signs were found in $71 \%$ (table 1). Four patients had an anterior opercular syndrome; details of these patients will be reported separately. Another patient presented with brief episodes of unconsciousness and asystole followed by a period of confusion. Her symptoms were attributed to heart disease and a cardiac pacemaker was inserted, but eventually it became clear that these attacks were complex partial seizures.

\section{NEUROIMAGING}

An area of decreased attenuation in one (17 patients) or both (eight patients) temporal lobes was found in 25 of the 38 patients $(66 \%)$ who had CT within 24 hours of starting acyclovir. The CT showed a mass effect in 11 patients $(29 \%)$, contrast enhancement in eight patients $(21 \%)$, and patchy haemorrhage in five patients (13\%). Only one patient had MRI at presentation; it showed a unilateral temporal lobe abnormality whereas, a few hours earlier, CT had been normal.

ELECTROENCEPHALOGRAPHY

Twenty eight patients had an EEG within 48 hours of starting treatment. The background activity showed a focal abnormality in 18 of 28 patients (64\%) and a symmetric generalised abnormality in eight of 28 patients (29\%). An 
Table 2 Clinical details of mortality after herpes simplex encephalitis (HSE)

\begin{tabular}{llll}
\hline $\begin{array}{l}\text { Age at } \\
\text { diagnosis }\end{array}$ & $\begin{array}{l}\text { Time from start of } \\
\text { treatment to death }\end{array}$ & Neurological signs before death & Cause of death \\
\hline 65 & 2 days & Coma & HSE \\
69 & 3 days & Coma, seizures & HSE \\
62 & 3 days & Coma & HSE \\
71 & 8 days & Coma & HSE \\
51 & 21 days & Coma, seizures & Staphylococcal septicaemia, bronchopneumonia \\
43 & 5 months & Severe amnesia, aphasia, anxiety, agitation & Metastatic melanoma \\
70 & 18 months & Persistent vegetative state & Bowel infarct \\
74 & 10 y & Severe deficit short term memory & Myocardial infarct \\
\hline
\end{tabular}

^ Died two days after completing treatment.

epileptiform abnormality was present in 21 of 28 patients $(75 \%)$ : periodic lateralised epileptiform discharges (PLEDs) in 14 patients $(50 \%)$, non-periodic focal epileptiform discharges in 10 patients $(36 \%)$, and focal electrographic seizures in seven patients $(25 \%)$. The epileptiform abnormalities were bilateral in five $(18 \%)$.

CEREBROSPINAL FLUID

The leucocyte count in the CSF ranged between 0 and $930 \times 10^{6} / 1$. Herpes simplex virus DNA was found in the first CSF specimen in all of the 36 patients in which this test was used. The virus was identified as HSV type 1 in 28 patients and HSV type 2 in three patients; in five patients CSF was not available for viral typing. The patients with HSV type 2 encephalitis were five months, 18 years, and 40 years old, and their presenting symptoms and signs did not differ from those with HSV type 1 encephalitis. Eleven patients with a positive polymerase chain reaction assay had a second lumbar puncture in the first week. In one of 11 patients HSV DNA had disappeared from the CSF at seven days, but it was still present in the other 10 . In the second week, eight of 17 repeat CSF specimens still contained HSV DNA. Beyond two weeks three of 10 patients still had a positive test; two of these patients had HSV type 2 encephalitis.

INITIAL DIAGNOSIS AND MANAGEMENT

After the initial assessment, the diagnosis was herpes simplex encephalitis in 16 patients $(38 \%)$, but other diagnoses were recorded in 24 patients $(57 \%)$. The CSF abnormalities were wrongly attributed to viral meningitis in four patients and partially treated bacterial meningitis in three. Other diagnoses included metabolic encephalopathy (five patients), stroke (four), cerebral abscess (two), viral respiratory tract infection (two), migraine (one), sinusitis (one), febrile convulsion of unknown cause (one), and cerebral malaria (one). An initial diagnosis was not recorded for two patients. The most common reason for failure to diagnose herpes simplex encephalitis was a delay in performing a lumbar puncture in patients with an acute confusional state, which was incorrectly attributed to a systemic infection.

Fifteen patients $(36 \%)$ were started on acyclovir on the day of admission, but treatment in the other 27 patients was delayed for up to 18 days. The duration of treatment in the 36 patients who completed the course of acyclovir was seven to 28 days (median 14 days). Three patients received a second course of acyclovir for a clinically suspected relapse five days, 12 days, and six weeks after initial treatment was stopped; the polymerase chain reaction assay was not repeated in these cases. A fourth patient had received acyclovir for 26 days, but HSV DNA was still detectable in the $\mathrm{CSF}$ at the end of treatment. Four months later neurological symptoms and a CSF pleocytosis recurred; HSV DNA was present in the CSF, but disappeared five days after restarting acyclovir.

\section{MORTALITY}

Four patients died during treatment and another person died two days after finishing acyclovir (table 2). Three patients had a severe persistent neurological deficit and died after a longer interval. The mortality rate was $12 \%$ at one month and $14 \%$ at six months.

HANDICAP AND QUALITY OF LIFE IN SURVIVORS Of the 34 survivors, two patients could not be traced, one patient declined assessment, and two patients were unable to travel to Auckland. There was enough information available from other sources to assess handicap and quality of life in these people. The 34 surviving patients were 13 months to 92 years old (median 45 years, mean 41 years) and were evaluated six months to 11 years (median 1.6 years, mean 2.6 years) after herpes simplex encephalitis. Eight patients were assessed in the first year.

Grades on the Glasgow outcome scale were grade 1 (dead within one month) in five patients $(12 \%)$, grade 2 (vegetative state) in one patient $(2 \%)$, grade 3 (severe disability) in seven patients $(17 \%)$, grade 4 (moderate disability) in nine patients (21\%), and grade 5 (good recovery) in 20 patients $(48 \%)$. Of the seven severely disabled patients, two had died after the first month; two were living at home, but had a profound impairment of anterograde memory and required constant supervision; one was severely confused, incontinent, dysphasic, and living in a private hospital; and two children had pronounced developmental delay and poorly controlled epilepsy. Of the nine moderately disabled (grade 4) patients, five were unemployed, two were working but had been unable to return to their original occupation, and two patients were still at school but had serious learning difficulties. Only one of the 20 grade 5 patients was asymptomatic and had a normal neurological examination. 
SYMPTOMS IN LONG TERM SURVIVORS

Of the 34 survivors, 29 were examined six months to 11 years after herpes simplex encephalitis; five of these patients were assessed in the first year. Two patients had premorbid conditions which affected the assessment: dyslexia (one patient), and epilepsy and homonymous hemianopia (one patient).

Memory impairment was reported by 20 of $29(69 \%)$ of the long term survivors. Forty five per cent of the patients had a personality or behavioural abnormality. Severe anxiety, impaired concentration, insomnia, irritability, fatigue, poor motivation, and emotional lability each were noted by $20 \%-35 \%$ of the patients. Five patients $(17 \%)$ had been treated for depression, including two who had attempted to commit suicide. Obsessional-compulsive behaviour (two patients), aggression (one), hyperphagia (one), and claustrophobia (one) were uncommon. None of these patients had a history of psychiatric illness before herpes simplex encephalitis.

Three patients were started on anticonvulsant treatment during the acute illness, but had been free of seizures after leaving hospital. Another patient had continued treatment for temporal lobe epilepsy that preceded the episode of herpes simplex encephalitis. The other five patients had poorly controlled simple or complex partial seizures and secondary generalised seizures despite anticonvulsant treatment. One patient was having simple partial seizures, but he was not receiving treatment.

Other symptoms were dysphasia (six patients), anosmia (six), dysarthria (five), dysphagia (three), and unilateral arm weakness (two).

NEUROLOGICAL SIGNS IN LONG TERM SURVIVORS Orientation in time and place and short term memory were evaluated in 27 of the 29 patients; the two children could not be assessed. Two patients $(7 \%)$ were disoriented in time and two patients $(7 \%)$ were disoriented in time and place. Orientation was normal in the others. Short term memory was normal in eight patients $(30 \%)$, mildly impaired in 13 patients $(48 \%)$ and severely affected in six patients $(22 \%)$. The score for the mini mental state examination was less than 25 in five of 27 $(18 \%)$ patients tested.

Unilateral or bilateral anosmia was found in $65 \%$ of the patients. Eleven had a mild non-fluent dysphasia, but only one had a severe global aphasia. Four patients had a pseudobulbar palsy secondary to an anterior opercular syndrome. Minor upper motor neuron signs (facial weakness, mild spasticity, or clumsiness in one arm) were noted in five patients. Two had a homonymous hemianopia, but in one this preceded herpes simplex encephalitis. One patient developed acute HSV retinitis two years after treatment for herpes simplex encephalitis.

PROGNOSTIC FACTORS

Development of stupor or coma before acyclovir was started (four of 29 good outcome, nine of 13 poor outcome, $\mathrm{P}<0.005)$ and older mean age (54 years poor outcome, 38 years good outcome, $\mathrm{P}<0.05$ ) were associated with a worse outcome. Of the eight patients who died, six were more than 60 years old when they developed herpes simplex encephalitis. The time from admission to the start of treatment was longer in the patients with a poor outcome (1.8 days good outcome, 4.0 days poor outcome, $\mathrm{P}<0.025)$, but the total duration of symptoms before starting treatment was not significantly different between the two groups (6.0 days good outcome, 7.7 days poor outcome, $\mathrm{P}<0.20)$. The frequency of seizures or focal neurological signs, the CSF leucocyte count, and CSF protein were not significantly different between patients with a good or poor outcome. Unilateral epileptiform abnormalities (PLEDs, non-periodic epileptiform discharges, and electrographic seizures) were not significantly different between the two groups, but bilateral epileptiform abnormalities were more common in patients with a poor outcome (0) of 18 good outcome, five of 10 poor outcome, $\mathrm{P}<0.01)$. An abnormal CT was also more common in patients with a poor outcome (11 of 11 patients) than in patients with a good outcome (14 of 27 patients) $(\mathrm{P}<0.025)$.

\section{Discussion}

Our study of the long term outcome after herpes simplex encephalitis differs from most of the other reports on the long term sequelae in several ways. (1) Rigorous criteria for the diagnosis of herpes simplex encephalitis were used. Before the development of the polymerase chain reaction assay for HSV DNA in 1989, some patients were treated with acyclovir without attempting to confirm the diagnosis of herpes simplex encephalitis with a brain biopsy or measurement of antibody in the CSF. Some of these patients probably had herpes simplex encephalitis, but they were not included in this study. (2) All patients were treated with acyclovir. (3) Patients were not selected for inclusion in the study because they had a known neurological or neuropsychological deficit.

Our study had several limitations. The study was retrospective and, consequently, patients were evaluated at a wide range of times after the acute illness. Most of our surviving patients were assessed more than one year after the acute illness, but eight people were evaluated after six to 12 months. Assessment within the first year, especially in the first few months, may not accurately reflect the long term outcome. $^{19}$

In our patients the six month mortality rate was similar to the acyclovir treated patients in the Swedish and American trials. ${ }^{112}$ In the long term about $30 \%$ of our patients either died or were severely disabled. Most survivors were living independently, but only one patient made a complete recovery. Other reports of small groups of patients with herpes simplex encephalitis also suggest that long term sequelae are common. Gordon et al assessed four patients one year or more after receiving acyclovir in the early stages of herpes simplex encephalitis. ${ }^{13}$ Their patients did not function at their prior level of achievement and had residual abnormalities on clinical or neuropsy- 
chological assessment. The most common findings were dysnomia and memory impairment. In another study, only one of 17 patients had a normal neuropsychological assessment two years or more after acyclovir treatment. ${ }^{14}$ Impairments in tests of learning, memory, general orientation and perceptual-motor skills were noted in the other patients. Dementia was seen in five people.

The most common and most disabling complication of herpes simplex encephalitis in our patients, and in other studies, ${ }^{9}{ }^{10} 20-24$ was memory impairment, which especially affected short term memory. Occasionally remote memory is more severely affected than anterograde memory. ${ }^{25}$ Memory impairment after herpes simplex encephalitis reflects the involvement of bilateral medial temporal lobe structures found in pathological, ${ }^{10} 2223$ imaging, ${ }^{20}$ and virological ${ }^{26}$ studies. Personality, behavioural, and psychiatric disorders occurred in almost half of our long term survivors, but the severe behavioural and emotional aberrations found before the introduction of acyclovir were uncommon. ${ }^{102427}$ Some survivors of herpes simplex encephalitis have developed the Kluver-Bucy syndrome, ${ }^{28}$ but we did not find this pattern in our patients.

Anosmia was found in two thirds of the evaluable surviving patients, although many of these people were unaware of the deficit. Although HSV may reach the brain by spreading along the olfactory nerves, ${ }^{26}$ anosmia has not been commonly reported after herpes simplex encephalitis. Epilepsy, mild dysphasia, and upper motor neuron signs were fairly common, but disabling focal neurological signs were infrequent.

The EEG is usually abnormal in herpes simplex encephalitis. ${ }^{29}{ }^{30}$ Two of our patients had a normal EEG, but we were unable to review these records. Abnormal background activity with a focal accentuation, PLEDs, and electrographic seizures are typical of herpes simplex encephalitis, but this constellation of findings is not specific for the diagnosis. ${ }^{29-31}$ The prognostic implications of the EEG in herpes simplex encephalitis are unclear. ${ }^{29}$ In other series of patients, unilateral ${ }^{32}{ }^{33}$ and bilateral ${ }^{34}$ PLEDs have been associated with a poor prognosis, but interpretation of these reports is limited by the variable interval between the EEG and the initiation of treatment, the few patients, and the unavailability of effective antiviral treatment. We only considered EEGs recorded at about the time treatment was started. All of our patients with bilateral epileptiform abnormalities had a poor long term outcome, but unilateral epileptiform abnormalities were not necessarily associated with a poor prognosis.

Computed tomography and MRI are often abnormal, but the findings are not specific for herpes simplex encephalitis. ${ }^{35}{ }^{36}$ Magnetic resonance imaging more commonly shows changes in the early stages of the illness and it has replaced CT as the imaging method of choice in patients with suspected herpes simplex encephalitis. ${ }^{36}$ The CT was abnormal in two thirds of our patients at the time of diagnosis.
In most of our patients the diagnosis of herpes simplex encephalitis was confirmed by finding HSV DNA in CSF. The polymerase chain reaction has provided a specific method of detecting HSV DNA in $\mathrm{CSF}^{1537}{ }^{38}$ and the sensitivity is similar to that of a brain biopsy. ${ }^{39}$ In our patients HSV DNA was always present in the first CSF specimen, but occasionally it is not detected in the first sample. ${ }^{37}{ }^{40}$ Herpes simplex virus DNA usually persisted in the CSF for one to two weeks after starting treatment and in some people, especially patients with HSV type 2 encephalitis, it lasted for longer. It is unclear if persistence of HSV DNA in CSF is a useful guide to the duration of treatment. Cinque et al recommended that acyclovir should be continued if HSV DNA is still present in the CSF after the first course of treatment. ${ }^{38}$ The polymerase chain reaction has provided a rapid, reliable, and less invasive test to confirm the diagnosis of herpes simplex encephalitis and, in most patients, brain biopsy is no longer necessary.

Although the polymerase chain reaction has facilitated the early diagnosis of herpes simplex encephalitis and acyclovir has reduced the mortality and long term morbidity, $30 \%$ of our patients still had a poor outcome. In our patients, poor outcome was associated with several factors that reflected delays in diagnosis: the development of stupor or coma before treatment, CT abnormalities, and a longer time between admission to hospital and the initiation of treatment. The total duration of symptoms before treatment was not significantly different between patients with poor and good outcomes, but the history may be unreliable in confused or unconscious patients. Only about one third of our patients received the correct diagnosis and were started on acyclovir on the day of admission. Further reduction of the mortality and morbidity of herpes simplex encephalitis in the future may be achieved with longer courses of treatment and new antiviral agents, but a key aim in improving the outcome should be a reduction in unnecessary delays in diagnosis and treatment.

We acknowledge the financial support of the June Byham Bequest, and the assistance of Chris Frampton and Michael Bequest, and the assistance of Chris $\mathrm{F}$
McCaskill in providing statistical advice.

1 Olson LC, Buescher EL, Artenstein MS, Parkman PD. Herpesvirus infections of the human central nervous system. $N$ Engl F Med 1967;277:1271-7.

2 Illis LS, Merry RTG. Treatment of herpes simplex encephalitis. FR Coll Physicians Lond 1972;7:34-44.

litis. $\mathcal{F} R$ Coll Physicians Lond 1972;7:34-44.
3 Whitley RJ, Soong S-J, Dolin R, et al. Adenine arabinoside Whitley RJ, Soong S-J, Dolin R, et al. Adenine arabinoside
therapy of biopsy-proved herpes simplex encephalitis. National Institute of Allergy and Infectious Diseases collaborative antiviral study. N Engl f Med 1977;297:28994.

4 Rennick PM, Nolan DC, Bauer RB, Lerner AM. Neuropsychologic and neurologic follow-up after herpesvirus hominis encephalitis. Neurology 1973;23:42-7

5 Sarubbi FA, Sparling PF, Glezen WP. Herpesvirus hominis encephalitis. Virus isolation from brain biopsy in seven patients and results of therapy. Arch Neurol 1973;29:26873.

6 Wolman B, Longson M. Herpes encephalitis. Acta Paediatr Scand $1977 ; 66: 243-6$

7 Williams BB, Lerner AM. Some previously unrecognized features of herpes simplex virus encephalitis. Neurology 1978;28:1193-6.

8 Kennedy PGE. A retrospective analysis of forty-six cases of herpes simplex encephalitis seen in Glasgow between 1962 and 1985. Q F Med 1988;68:533-40.

9 Oxbury JM, MacCallum FO. Herpes simplex virus encephalitis: clinical features and residual damage. Postgrad Med f 1973;49:387-9. 
10 Hierons R, Janota I, Corsellis JAN. The late effects of necrotizing encephalitis of the temporal lobes and limbic 1978;8:21-42.

11 Skoldenberg B, Forsgren M, Alestig K, et al. Acyclovir versus vidarabine in herpes simplex encephalitis. Randomised multicentre study in consecutive Swedish patients. Lancet 1984;ii:707-11

12 Whitley RJ, Alford CA, Hirsch MS, et al. Vidarabine versus acyclovir therapy in herpes simplex encephalitis. $N$ Engl $\mathcal{F}$ Med 1986;314:144-9.

13 Gordon B, Selnes OA, Hart J, Hanley DF, Whitley RJ Long-term cognitive sequelae of acyclovir-treated herpes simplex encephalitis. Arch Neurol 1990;47:646-7.

14 Skoldenberg B. Herpes simplex encephalitis. Scand f Infect Dis 1991; (suppl 80):40-6.

15 Anderson NE, Powell KF, Croxson MC. A polymerase chain reaction assay of cerebrospinal fluid in patients with chain reaction assay of cerebrospinal fluid in patients with
suspected herpes simplex encephalitis. $\mathcal{F}$ Neurol Neurosurg Psychiatry 1993;56:520-5.

16 Folstein MF, Folstein SE, McHugh PR. "Mini-mental state": a practical method for grading the cognitive state of state": a practical method for grading the cognitive state of
patients for the clinician. $\mathcal{F}$ Psychiatr Res $1975 ; 12: 189-98$.

17 Jennett B, Bond $M$. Assessment of outcome after severe brain damage. A practical scale. Lancet 1975; i:480-4

18 Jennett B, Snoek J, Bond MR, Brooks N. Disability after severe head injury: observations on the use of the Glasgow outcome scale. F Neurol Neurosurg Psychiatry 1981;44:28593.

19 Booss J, Esiri MM. Sporadic encephalitis I. In: Booss J, Esiri MM, eds. Viral encephalitis. Pathology, diagnosis and management. Oxford: Blackwell, 1986:55-93.

20 Kapur N, Barker S, Burrows EH, et al. Herpes simplex encephalitis: long term magnetic resonance imaging and neuropsychological profile. $\mathcal{F}$ Neurol Neurosurg Psychiatry 1994;57:1334-42.

21 Rose FC, Symonds CP. Persistent memory defect following encephalitis. Brain 1960;83:195-212.

22 Drachman DA, Adams RD. Herpes simplex and acute inclusion-body encephalitis. Arch Neurol 1962;7:45-63.

23 Friedman HM, Allen N. Chronic effects of complete limbic lobe destruction in man. Neurology 1969;19:679-90.

24 Greenwood R, Bhalla A, Gordon A, Roberts J. Behaviour disturbances during recovery from herpes simplex encephalitis. F Neurol Neurosurg Psychiatry 1983;46:809-17.

25 O'Connor M, Butters N, Miliotis P, Eslinger P, Cermak LS The dissociation of anterograde and retrograde amnesia in a patient with herpes encephalitis. F Clin Exp Neuropsychol 1992;14:159-78.

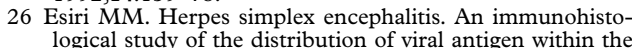
brain. F Neurol Sci 1982;54:209-26.
27 Laurent B, Allegri RF, Thomas-Anterion C, Foyatier N, Naegele-Faure B, Pellat J. Long term neuropsychological follow-up in patients with herpes simplex encephalitis and predominantly left-sided lesions. Behavioural Neurology 1991;4:211-24

28 Hart RP, Kwentus JA, Frazier RB, Hormel TL. Natural history of Kluver-Bucy syndrome after treated herpes encephalitis. South Med f 1986;79:1376-8

29 Lai CW, Gragasin ME. Electroencephalography in herpes simplex encephalitis. f Clin Neurophysiol 1988;5:87-103.

30 Brick JF, Brick JE, Morgan JJ, Gutierrez AR. EEG and pathologic findings in patients undergoing brain biopsy for suspected encephalitis. Electroencephalogr Clin Neurophysiol 1990;76:86-9.

31 Illis LS, Taylor FM. The electroencephalogram in herpessimplex encephalitis. Lancet 1972;i:718-21.

32 Elian M. Herpes simplex encephalitis. Prognosis and long-term follow-up. Arch Neurol 1975;32:39-43.

33 Dutt MK, Johnston IDA. Computed tomography and EEG in herpes simplex encephalitis. Their value in diagnosis and prognosis. Arch Neurol 1982;39:99-102.

34 Brodtkorb E, Lindqvist M, Jonsson M, Gustafsson A. Diagnosis of herpes simplex encephalitis. A comparison between electroencephalography and computed tomography findings. Acta Neurol Scand 1982;66:462-71.

35 Davis JM, Davis KR, Kleinman GM, Kirchner HS, Taveras JM. Computed tomography of herpes simplex encephalitis, with clinicopathological correlation. Radiology 1978;129: 409-17.

36 Tien RD, Felsberg GJ, Osumi AK. Herpesvirus infections of the CNS: MR findings. Am f Roentgenol 1993;161:167-76.

37 Aurelius E, Johansson B, Skoldenberg B, Staland A, Forsgren $M$. Rapid diagnosis of herpes simplex encephalitis by nested polymerase chain reaction assay of cerebrospinal fluid. Lancet 1991;337:189-92.

38 Cinque $\mathrm{P}$, Cleator GM, Weber $\mathrm{T}$, et al. The role of laboratory investigation in the diagnosis and management of patients with suspected herpes simplex encephalitis: a consensus report. F Neurol Neurosurg Psychiatry 1996;61: 339-45.

39 Lakeman FD, Whitley RJ, and the National Institute of Allergy and Infectious Diseases Collaborative Antiviral Study Group. Diagnosis of herpes simplex encephalitis: Application of polymerase chain reaction to cerebrospinal fluid from brain-biopsied patients and correlation with disease. F Infect Dis 1995;171:857-63.

40 Rozenberg F, Lebon P. Amplification and characterization of herpesvirus DNA in cerebrospinal fluid from patients with acute encephalitis. $\mathcal{F}$ Clin Microbiol 1991;29:2412-7. 